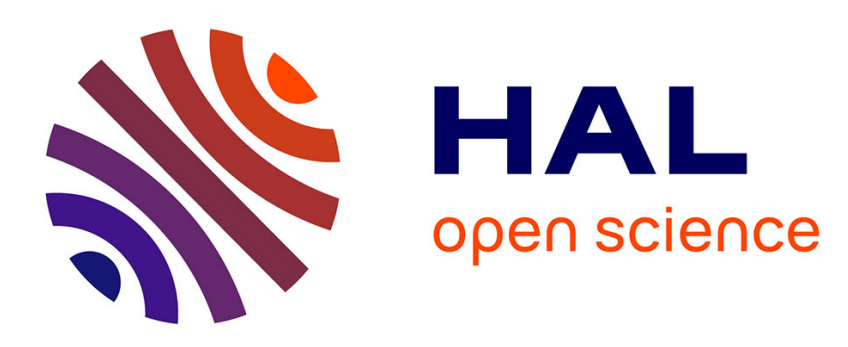

\title{
Generic Linear ESO for the State Observation of Unknown Nonlinear SISO Systems
}

Fawzia Amokrane, Emmanuel Piat, Joël Abadie, Adrien Drouot, Juan Antonio Escareno

\section{- To cite this version:}

Fawzia Amokrane, Emmanuel Piat, Joël Abadie, Adrien Drouot, Juan Antonio Escareno. Generic Linear ESO for the State Observation of Unknown Nonlinear SISO Systems. International Conference on MechatrOnics, Robotics, and System Engineering, Dec 2019, Bali, Indonesia. 10.1109/MoRSE48060.2019.8998678 . hal-03053032

\section{HAL Id: hal-03053032 https://hal.science/hal-03053032}

Submitted on 10 Dec 2020

HAL is a multi-disciplinary open access archive for the deposit and dissemination of scientific research documents, whether they are published or not. The documents may come from teaching and research institutions in France or abroad, or from public or private research centers.
L'archive ouverte pluridisciplinaire HAL, est destinée au dépôt et à la diffusion de documents scientifiques de niveau recherche, publiés ou non, émanant des établissements d'enseignement et de recherche français ou étrangers, des laboratoires publics ou privés. 


\section{Generic Linear ESO for the State Observation of Unknown Nonlinear SISO Systems}

\author{
$1^{\text {st }}$ Fawzia Amokrane \\ FEMTO-ST Institute, Univ. Bourgogne \\ Franche-Comté, CNRS UMR 6174 \\ 25000 Besançon, France \\ fawzia.amokrane@femto-st.fr
}

\author{
$2^{\text {nd }}$ Emmanuel Piat \\ FEMTO-ST Institute, Univ. Bourgogne \\ Franche-Comté, CNRS UMR 6174 \\ 25000 Besançon, France \\ emmanuel.piat@ens2m.fr
}

\author{
$3^{\text {rd }}$ Joël Abadie \\ FEMTO-ST Institute, Univ. Bourgogne \\ Franche-Comté, CNRS UMR 6174 \\ 25000 Besançon, France \\ joel.abadie@femto-st.fr
}

\author{
$4^{\text {th }}$ Adrien Drouot \\ FEMTO-ST Institute, Univ. Bourgogne \\ Franche-Comté, CNRS UMR 6174 \\ 25000 Besançon, France \\ adrien.drouot@femto-st.fr
}

\author{
$5^{\text {th }}$ Juan Escareno \\ XLIM, Univ. Limoges, CNRS UMR 7252 \\ 87000 Limoges, France \\ juan.escareno-castro@unilim.fr
}

\begin{abstract}
This paper introduces a generic procedure for the state estimation of unknown nonlinear SISO systems, i.e. when no information is available on their structure, possibly time-varying parameters and potential disturbances. Such systems are met for instances for systems based on complex micro and nano mechatronic designs that are interacting in an unknown way with their environment at nano scales. This procedure relies on the choice of an arbitrary linear model and the use of a Generic Linear Extended State Observer, whose principle is also introduced in the paper. The proposed approach overcomes wellknown model-based nonlinear techniques in the sense that it is easy to implement, all the while avoiding any identification step and mathematical complexity. Simulation results involving nonlinear systems, subject to external disturbances, compare the performance of the proposed approach to the one of some modelfree nonlinear observers described in the literature.
\end{abstract}

Index Terms - Observer for Unknown Nonlinear Systems, Extended State Observer, ADRC, Micro Mechatronic Systems

\section{INTRODUCTION}

Owing to its importance in modern control theory, the field of state observation for dynamical systems has been an active area of research for decades. Based only on the inputs and outputs of any given system, the resulting observers are expected to produce an estimation of the states, that is then used by the control structure. For linear systems, one can cite the works of Kalman for stochastic systems [1] and Luenberger for deterministic systems [2]. However, physical systems may feature some unexpected complexity due to, e.g., inherent nonlinearities, unavoidable and unknown changes of their structure (uncertainties), the influence of the environment (disturbances), etc. Therefore, these systems are no longer linear and that is why many theoretical and practical developments focus on the design of nonlinear observers.

There exist various strategies for the design of observers for nonlinear systems. There are, for example, the extended

This work has been supported by the EIPHI Graduate School, contract ANR-17-EURE-0002.
Kalman filter or one of its many extensions [3], Lie algebrabased observers [4], optimization-based observers [5], highgain observers [6], etc. Of all of the above approaches, the high-gain observers have received the most attention due to their simplicity and good performance in noise-free settings [6]. Indeed, in the high-gain observers paradigm, the estimation error trajectory has an exponential decay rate that can be chosen arbitrarily fast by acting on a design parameter that appears in the observers structure. Nevertheless, the highgain observers design also highlights drawbacks, including implementation issues due to the value of the design parameter, the peaking phenomenon during the transient and a sensitivity to measurement noise [7]. Recent works alleviated these undesirable properties [8], but this has been achieved at the cost of a more complicated design, i.e. increase of the observers dimension to $2 n-1$, where $n$ is the order of the system, use of saturation functions in the observers dynamics, etc.

Furthermore, a major limitation that is common to all the above approaches is that they all require some a priori information on the structure of the systems, e.g. their order, a Lipschitzian behavior [6], [8], a stable zero dynamics [9], etc. However in many industrial cases involving micro and nano mechatronic designs, some parameters may be affected by large uncertainties and cannot be easily measured (e.g. mass, inertia, etc.) nor identified, but these systems still require efficient observation and control schemes. Also, as the mathematical complexity inherent to the nonlinear nature of the systems may be an issue, there is a growing interest in the development of accessible and general methods to solve the problem of state observation and control of such unknown nonlinear systems. Owing to its less dependence on systems information, its abilities to cope with a wide range of uncertainties and disturbances, and its simplicity in the control structure, the Active Disturbance Rejection Control (ADRC) framework [10]-[12] is a significant step towards this purpose. 
The idea of ADRC consists in estimating both the state and a total disturbance, that lumps unmodeled dynamics and external disturbances into an extended state, by an Extended State Observer (ESO) [13]. Thus, the state of the unknown systems becomes available for control purposes and the total disturbance can be compensated for in real time. Following the ESO parameterization steps described in [14], the ADRC technique has been used to solve various kinds of mechatronic problems, e.g., motor control [15], flight control [16], robot control [17], etc. Yet, many of the ESO developed in the literature like the Standard Linear Extended State Observer (SLESO) [17] [19] are of order $n+1$. This implicitly assumes that the total disturbance is constant or slowly time-varying. Therefore, in the common case of non-constant total disturbance, the quality of the estimation provided by the standard ESO becomes insufficient [20]. In order to improve the efficiency of the ESO, their order has to be increased and that idea paved the way to the design of higher-order ESO [20]-[22], Generalized Proportional Integral observers (GPI) [23], [24], etc. Indeed, increasing the number of extended states allows to effectively reconstruct a total disturbance described by complex and sophisticated high order polynomial.

This paper addresses the on-line state observation problem of a specific class of nonlinear SISO systems that are totally unknown. In the proposed procedure, the unknown nonlinear system is represented as a chosen (arbitrarily because no information is provided) linear system to which is added an unknown non physical exogenous input, called a virtual input. That exogenous input can be seen as the total disturbance of the ADRC framework as it gathers all the neglected nonlinearities, unmodeled dynamics, parameter uncertainties, and external disturbances such that the input-output dynamics of the linear system matches the one of the nonlinear system. Then, extending the state of the linear system with that unknown virtual input, its behavior can be estimated with any linear extended state observation technique, along with the state of the system. In this paper, this will be achieved by a Generic Linear Extended State Observer (GeLESO). This new concept is a less conservative version of higher-order ESO and GPI observers used in ADRC in the sense that the GeLESO is built upon a linear system whose features, i.e. order, bandwidth, etc., are chosen and therefore not unique. Furthermore, the order of this observer can be increased at will in order to preserve the estimation accuracy even with unknown complex virtual input. In the following, the design procedure of the GeLESO is described, its performance is validated by simulations and its behavior is compared to higher-order ESO / GPI observers.

\section{Problem StATEMEnT}

This paper addresses the state observation problem of unknown nonlinear and time-varying SISO systems whose inputoutput dynamics can be described by an Ordinary Differential Equation (ODE) of the form:

$$
y^{(n)}(t)=f\left(y(t), \ldots, y^{(n-1)}(t), u(t), U(t), t\right)
$$

where

- the order $n>0$ of the ODE is unknown;

- the nonlinear and time-varying function $f$ exists, but is unknown;

- the scalar $y(t) \in \mathbb{R}$ is the output of the system that is provided to the observer. It is known, but its $n$ successive derivatives are unknown;

- the scalar $u(t) \in \mathbb{R}$ is the output of the controller that is also provided to the observer, hence it is known;

- the input $U(t) \in \mathbb{R}^{\delta}$, where $\delta$ is unknown, represents the uncertainties and disturbances that can affect the system in any way, hence it is unknown.

The state vector $\mathcal{X}(t) \in \mathbb{R}^{n}$ of system (1) is defined by:

$$
\mathcal{X}(t)=\left[\begin{array}{llll}
y(t) & \dot{y}(t) & \ldots & y^{(n-1)}(t)
\end{array}\right]^{\top}
$$

where only its first component $y(t)$ is known.

Let's now assume that, by the designer's choice, the first $p$ components of $\mathcal{X}(t)$ are actually necessary to implement the control algorithm of the unknown system (1). These $p$ components, gathered in the vector

$$
X(t)=\left[\begin{array}{llll}
y(t) & \dot{y}(t) & \ldots & y^{(p-1)}(t)
\end{array}\right]^{\top} \in \mathbb{R}^{p},
$$

have therefore to be estimated despite the fact that no information is available on the system. This is the point of the paper.

Note that, as the order $n$ of the system is unknown, $p$ can unknowingly end up less, equal or greater than $n$. In the first two cases, the methodology introduced in this paper would respectively lead to a reduced-order or a full-order observer of $\mathcal{X}(t)$. In the last case, which will not be addressed in this paper due to a lack of relevance, the designed observer would estimate the $n$ components of $\mathcal{X}(t)$ along with the $p-n$ following successive derivatives of the output.

\section{EQUIVALENT STATE-SPACE REPRESENTATION}

In this section, it is shown that the nonlinear ODE of order $n$ (1) can be described by an equivalent linear ODE of order $p$. Indeed, (1) can be written as:

$$
\begin{aligned}
& y^{(n)}-f\left(y, \ldots, y^{(n-1)}, u, U, t\right) \\
& -y^{(p)}+\left(a_{1} y+\ldots+a_{p} y^{(p-1)}+b u\right) \\
& +y^{(p)}-\left(a_{1} y+\ldots+a_{p} y^{(p-1)}+b u\right)=0
\end{aligned}
$$

where the coefficients $a_{k} \in \mathbb{R}$, with $k=1, \ldots, p$, and $b \in$ $\mathbb{R}^{*}$ are chosen.

Let's now define a virtual input $\mathscr{C} \in \mathbb{R}$ as:

$$
\begin{aligned}
\mathscr{C} \triangleq & -\frac{1}{b}\left[y^{(n)}-f\left(y, \ldots, y^{(n-1)}, u, U, t\right)\right. \\
& \left.-y^{(p)}+\left(a_{1} y+\ldots+a_{p} y^{(p-1)}+b u\right)\right]
\end{aligned}
$$

such that (4) becomes equivalent to the following ODE of order $p$ :

$$
y^{(p)}=a_{1} y+\ldots+a_{p} y^{(p-1)}+b u+b \mathscr{C} .
$$




$$
y^{(p)}=a_{1} y+\ldots+a_{p} y^{(p-1)}+b u+b \mathscr{C} .
$$

As both (1) and (7) have the same first $p$ initial conditions $y\left(t_{0}\right), \ldots, y^{(p-1)}\left(t_{0}\right),(7)$ has the same input-output behavior as (1).

Combining (3) and (7) leads to the following state-space representation:

$$
\left\{\begin{aligned}
\dot{X} & =A X+B u+B \mathscr{C} \\
y & =C X
\end{aligned}\right.
$$

where the matrices $A \in \mathbb{R}^{p \times p}, B \in \mathbb{R}^{p \times 1}$ and $C \in \mathbb{R}^{1 \times p}$ are given by:

$$
\begin{gathered}
A=\left[\begin{array}{cc}
\mathbf{0}_{(p-1) \times 1} & I_{p-1} \\
a_{1} & \mathscr{A}
\end{array}\right] \text { with } \mathscr{A}=\left[\begin{array}{lll}
a_{2} & \ldots & a_{p}
\end{array}\right] \\
B=\left[\begin{array}{c}
\mathbf{0}_{(p-1) \times 1} \\
b
\end{array}\right] \text { and } C=\left[\begin{array}{ll}
1 & \mathbf{0}_{1 \times(p-1)}
\end{array}\right] .
\end{gathered}
$$

with 0 standing for zero matrices of appropriate dimensions.

As a result, the state estimation problem of the unknown nonlinear system (1) becomes equivalent to the state estimation of the known linear system (8), combined with the estimation of the unknown virtual input $\mathscr{C}$.

\section{Generic Linear Extended State Observer}

The Generic Linear Extended State Observer (GeLESO), able to estimate not only the state $X$ of the system, but also the virtual input $\mathscr{C}$, is introduced in this section.

\section{A. Design of the GeLESO}

As most approaches in the literature, this new observation scheme relies on the incorporation of the virtual input $\mathscr{C}$ into the state $X$ to form an extended state $X_{e}$. However unlike the usual approaches, the GeLESO will ingeniously use the information provided by the $p$ coefficients $a_{k}$ and by the successive derivatives of $\mathscr{C}$. It is designed as follows:

Let $i \in \mathbb{N}^{*}$ be the chosen number of extended components in the extended state $X_{e}$ such that:

$$
X_{e}=\left[\begin{array}{llllll}
x_{1} & \ldots & x_{p} & x_{p+1} & \ldots & x_{p+i}
\end{array}\right]^{\top} \in \mathbb{R}^{p+i}
$$

and let's assume that the virtual input $\mathscr{C}$ is of class $C^{m}$, with $m \in \mathbb{N}^{*}$ and $m \geq i$.

The key idea in the proposed observation scheme is to introduce a state component into $X_{e}$ for each derivative of $\mathscr{C}$ to be estimated. This new component is based on the time derivative of the previous one, where all the components are kept, but the last one. This leads to:

$$
\left\{\begin{aligned}
\dot{x}_{1} & =x_{2} \\
& \vdots \\
\dot{x}_{p} & =x_{p+1}+a_{p} x_{p}+b u \\
\dot{x}_{p+1} & =x_{p+2}+a_{p-1} x_{p} \\
& \vdots \\
\dot{x}_{2 p-1} & =x_{2 p}+a_{1} x_{p} \\
\dot{x}_{2 p} & =b \mathscr{C}^{(p)} \\
& \vdots \\
\dot{x}_{p+i} & =b \mathscr{C}^{(i)} \\
y & =x_{1}
\end{aligned}\right.
$$

with (as an example of the design procedure)

$$
x_{p+1}=a_{1} x_{1}+a_{2} x_{2}+\ldots+a_{p-1} x_{p-1}+b \mathscr{C}
$$

and where the first $p$ rows represent system (8). The following $p-1$ rows, i.e. from $\dot{x}_{p+1}$ to $\dot{x}_{2 p-1}$, represent the dynamics of the extended state components introduced as aforementioned. These rows correspond to the case where, by choice, $1 \leq i<p$. The final rows, i.e. from $\dot{x}_{2 p}$ to $\dot{x}_{p+i}$, represent the dynamics of the components subsequently introduced, and only composed of the successive derivatives of $\mathscr{C}$. Those rows only exist in the case where $p \leq i \leq m$. The Generic Linear Extended State Observer estimating the extended state $X_{e}$, hence giving both the state $X$ and the virtual input $\mathscr{C}$, is built upon (11) and is given by:

$$
\left\{\begin{aligned}
\dot{z}_{1} & =z_{2}+L_{1}\left(y-z_{1}\right), \\
& \vdots \\
\dot{z}_{p} & =z_{p+1}+a_{p} z_{p}+L_{p}\left(y-z_{1}\right)+b u \\
\dot{z}_{p+1} & =z_{p+2}+a_{p-1} z_{p}+L_{p+1}\left(y-z_{1}\right), \\
& \vdots \\
\dot{z}_{2 p-1} & =z_{2 p}+a_{1} z_{p}+L_{2 p-1}\left(y-z_{1}\right), \\
\dot{z}_{2 p} & =z_{2 p+1}+L_{2 p}\left(y-z_{1}\right), \\
& \vdots \\
\dot{z}_{p+i} & =L_{p+i}\left(y-z_{1}\right)
\end{aligned}\right.
$$

where $z_{1}, \ldots, z_{p+i}$ are the $p+i$ components of the observer's state $Z_{e}$, and where $L_{1}, \ldots, L_{p+i}$ are the gains of the observer.

Substracting (13) from (11) leads to the dynamics of the estimation error $E$ that reads as follows:

$$
\dot{E}=\dot{X}_{e}-\dot{Z}_{e}=\left(A_{e}-\mathcal{L} C_{e}\right) E+B_{e} \mathscr{C}^{(i)}
$$

where the matrices $A_{e} \in \mathbb{R}^{(p+i) \times(p+i)}, \mathcal{L} \in \mathbb{R}^{(p+i) \times 1}, C_{e} \in$ $\mathbb{R}^{1 \times(p+i)}$ and $B_{e} \in \mathbb{R}^{(p+i) \times 1}$ are respectively given by:

$$
\begin{aligned}
& A_{e}=\left[\begin{array}{c|c|c}
\mathbf{0}_{(p+i-1) \times 1} & I_{p-1} & \mathbf{0}_{(p-1) \times i} \\
\cline { 2 - 3 } & \mathscr{A}^{\prime} & I_{i} \\
\hline \multicolumn{2}{c}{\mathbf{0}_{1 \times(p+i)}}
\end{array},\right. \\
& \mathcal{L}=\left[\begin{array}{llllllllll}
L_{1} & \ldots & L_{p} & L_{p+1} & \ldots & L_{2 p-1} & L_{2 p} & \ldots & L_{p+i}
\end{array}\right]^{\top}, \\
& C_{e}=\left[\begin{array}{ll}
1 & \mathbf{0}_{1 \times(p+i-1)}
\end{array}\right] \quad \text { and } B_{e}=\left[\begin{array}{ll}
\mathbf{0}_{(p+i-1) \times 1} & b
\end{array}\right]^{T}
\end{aligned}
$$

where $\mathbf{0}$ stands for zero matrices of appropriate dimensions, and where

$$
\mathscr{A}^{\prime}=\left[a_{p}, \ldots, a_{1}, \mathbf{0}_{(i-p) \times 1}\right]^{T}, \quad \text { if } p \leq 2
$$

or

$$
\mathscr{A}^{\prime}=\left[\begin{array}{c|c}
\mathbf{0}_{i \times(p-2)} & a_{p} \\
\vdots \\
a_{1} \\
\mathbf{0}_{(i-p) \times 1}
\end{array}\right], \quad \text { if } p>2 .
$$

\section{B. Tuning of the GeLESO}

Provided that the pair $\left(A_{e}, C_{e}\right)$ is observable, the gains matrix $\mathcal{L}$ in (14) must be chosen such that the matrix $\mathcal{A}=$ $A_{e}-\mathcal{L} C_{e}$ is Hurwitz. This can be done using pole placement techniques, linear quadratic routines or any other optimization 
design. In this paper, in order to ease the tuning of the observer and following the steps of [14] and [25], a single parameter $\omega_{o}>0$ is used to specify the value of the gains in $\mathcal{L}$. They are defined as follows:

$$
\begin{aligned}
\mathcal{L} & =\left[\begin{array}{llll}
L_{1} & L_{2} & \ldots & L_{p+i}
\end{array}\right]^{\top} \\
& =\left[\begin{array}{llll}
\gamma_{1} \omega_{o} & \gamma_{2} \omega_{o}^{2} & \ldots & \gamma_{p+i} \omega_{o}^{p+i}
\end{array}\right]^{\top}
\end{aligned}
$$

where

$$
\gamma_{j}=\frac{(p+i) !}{j !(p+i-j) !}, \quad \text { with } j=1, \ldots, p+i .
$$

With such a choice of gains, it has been shown in [25] that the observer's poles are all located in a close neighborhood of $-\omega_{o}$ provided that the parameter $\omega_{o}$ is chosen large enough, hence leading $\mathcal{A}$ to be Hurwitz.

\section{Convergence of the GeLESO}

Let's assume that $\mathscr{C}^{(i)}$ in (14) is bounded by some positive constant $\beta$, i.e. $\forall i \in \mathbb{N}^{*}, \exists \beta \in \mathbb{R}^{+}$such that $\left|\mathscr{C}^{(i)}\right| \leq \beta$. The convergence of the proposed Generic Linear Extended State Observer is then established in the following theorem:

Theorem 1 (convergence of the GeLESO): $\forall p \in \mathbb{N}^{*}$, order of the linear system (8); $\forall m \in \mathbb{N}^{*}$, the class of the virtual input $\mathscr{C} ; \forall i \in \mathbb{N}^{*}$, the chosen number of extended components in the extended state $X_{e}$ with $i \leq m$ and $\forall j=1, \ldots, p+i$, the following statements are true:

$$
\begin{aligned}
& \mathbb{S} 1: \text { if } \mathscr{C}^{(i)}=0 \text {, then } \lim _{t \rightarrow \infty} E(t)=0 \\
& \mathbb{S} 2: \text { if } \mathscr{C}^{(i)} \neq 0 \text {, then } \\
& \quad \exists \alpha \in \mathbb{R}^{+} \text {such that } \lim _{t \rightarrow \infty}\left|E_{j}(t)\right| \leq \alpha
\end{aligned}
$$

Proof: For reason of space, the proof of this theorem is omitted but it can be found in [26].

Statement $\mathbb{S} 1$ claims, when the last derivative of $\mathscr{C}$ considered is null, that all the components of the estimation error $E$ converge to 0 . On the other hand, statement $\mathbb{S} 2$ states, when the last derivative of $\mathscr{C}$ considered is different from 0 , that each component of the estimation error $E$ is bounded, the latter being provided in the proof in [26].

\section{Order of the GeLESO}

Let's consider two Generic Linear Extended State Observers, designed with a different order, but with the same parameter $\omega_{o}$ chosen large enough (see the proof of Theorem 2 in Appendix). The one of order $p+i$ will be noted GeLESO $\left.\right|_{i}$ and the one of order $p+k$ will be noted GeLESO $\left.\right|_{k}$. The influence of the order of the GeLESO on the estimation error is established in the following theorem:

Theorem 2 (order of the GeLESO): Provided that $\omega_{o}$ is chosen large enough (see the proof in Appendix), $\forall p \in \mathbb{N}^{*}$, order of the linear system (8); $\forall i \in \mathbb{N}^{*}$, the chosen number of extended components in the extended state $\left.X_{e}\right|_{i}$ of the GeLESO $\left.\right|_{i} ; \forall k \in \mathbb{N}^{*}$, the chosen number of extended components in the extended state $\left.X_{e}\right|_{k}$ of the GeLESO $\left.\right|_{k}$ with $k>i$ and $\forall j=1, \ldots, p+i$ :

- if $\mathscr{C}^{(i)}=0$, i.e. statement $\mathbb{S} 1$ of Theorem 1 , then

$$
\left.\lim _{t \rightarrow \infty} E(t)\right|_{k}=\left.\lim _{t \rightarrow \infty} E(t)\right|_{i}=0
$$

- if $\mathscr{C}^{(i)} \neq 0$, i.e. statement $\mathbb{S} 2$ of Theorem 1 , then

$$
\left.\lim _{t \rightarrow \infty}\left|E_{j}(t)\right|\right|_{k}<\left.\left.\lim _{t \rightarrow \infty}\left|E_{j}(t)\right|\right|_{i} \Leftrightarrow \alpha\right|_{k}<\left.\alpha\right|_{i}
$$

Proof: For reason of space, the proof of this theorem is omitted but it can be found in [26].

This theorem states, in the case where the estimation error does not converge to 0 , that the more the order of the GeLESO increases, the more the estimation error $E(t)$ on the extended state $X_{e}$ decreases.

\section{Simulation Results}

In order to illustrate its efficiency, higher-order GLESO is compared to higher-order ESO [20], [21] and GPI observers [23], [24]. The three approaches are used to estimate the state of the following system whose parameters are supposed unknown, like for some micro mechatronic systems:

$$
\left\{\begin{aligned}
\dot{x}_{1} & =x_{2}, \\
\dot{x}_{2} & =x_{3}, \\
\dot{x}_{3} & =-5 \theta x_{1}-2.92 x_{2}-\theta x_{3}+x_{1}^{2}+d, \\
y & =x_{1}
\end{aligned}\right.
$$

where $\theta$ is an uncertain parameter and $\mathcal{X}=\left[\begin{array}{lll}x_{1} & x_{2} & x_{3}\end{array}\right]^{\top}$ is the unknown state of the system, $y$ is its output that is known and $d$, with $d(t)=\sin (2 t)$, is an unknown disturbance. To preserve the coherence of the comparison, the parameters and the initial conditions of (18) are kept the same as in [23], i.e. $\theta=1.2, x_{1}(0)=-1, x_{2}(0)=-2$ and $x_{3}(0)=1$.

The first step of the design procedure is to define the chosen linear system (8), of chosen order $p>0$, equivalent to (18). The choice of $p$ relies on the controller's requirements and will be set to 3 in this example. According to (9), matrices $A$, $B$ and $C$ are given by:

$$
A=\left[\begin{array}{ccc}
0 & 1 & 0 \\
0 & 0 & 1 \\
a_{1} & a_{2} & a_{3}
\end{array}\right], \quad B=\left[\begin{array}{l}
0 \\
0 \\
b
\end{array}\right] \quad \text { and } C=\left[\begin{array}{lll}
1 & 0 & 0
\end{array}\right]
$$

where, by choice, $a_{1}=-8, a_{2}=-8, a_{3}=-0.8$ and $b=1$.

The second step of the procedure is to choose $i \in \mathbb{N}^{*}$, the number of extended components in the extended state $X_{e}(10)$. Still to preserve the coherence of the comparison, $i$ is set to 5 as it was done in [23]. With that choice, the structure of the GeLESO (13), the GPI observer [23], [24] and the higherorder ESO [20], [21] are given in Table I, where $e_{1}=y-z_{1}$.

In addition, the initial conditions of the three observation schemes are all null and their observation gains $L_{1}, \ldots, L_{8}$ are computed using (16) and (17), with $\omega_{o}=5$. With these settings, and because they have the same internal structure, the 
TABLE I

STRUCTURE OF THE DIFFERENT OBSERVERS

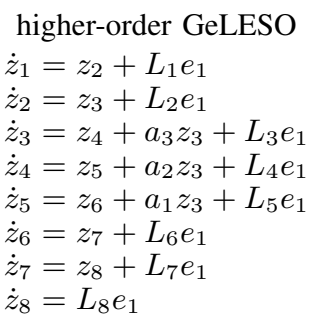

GPI observer

higher-order ESO

$\dot{z}_{1}=z_{2}+L_{1} e_{1}$

$\dot{z}_{1}=z_{2}+L_{1} e_{1}$

$\dot{z}_{2}=z_{3}+L_{2} e_{1} \quad \dot{z}_{2}=z_{3}+L_{2} e_{1}$

$\dot{z}_{3}=\rho_{1}+L_{3} e_{1} \quad \dot{z}_{3}=z_{4}+L_{3} e_{1}$

$\dot{\rho}_{1}=\rho_{2}+L_{4} e_{1} \quad \dot{z}_{4}=z_{5}+L_{4} e_{1}$

$\dot{\rho}_{2}=\rho_{3}+L_{5} e_{1} \quad \dot{z}_{5}=z_{6}+L_{5} e_{1}$

$\dot{\rho}_{3}=\rho_{4}+L_{6} e_{1} \quad \dot{z}_{6}=z_{7}+L_{6} e_{1}$

$\dot{\rho}_{4}=\rho_{5}+L_{7} e_{1} \quad \dot{z}_{7}=z_{8}+L_{7} e_{1}$

$\dot{\rho}_{5}=L_{8} e_{1} \quad \dot{z}_{8}=L_{8} e_{1}$
GPI observer and the higher-order ESO will provide the exact same estimation of $x_{1}, x_{2}$ and $x_{3}$.

Figure 1 illustrates that (18) (black curve) gives the same temporal behaviour $y(t)$ as (8) (blue curve). The virtual input $\mathscr{C}$ plotted in red is computed using (5) with the coefficients $a_{i}$ defined above (19), its estimation is in the dashed blue line. Note that the peak in the transient phase of the estimation is cropped.
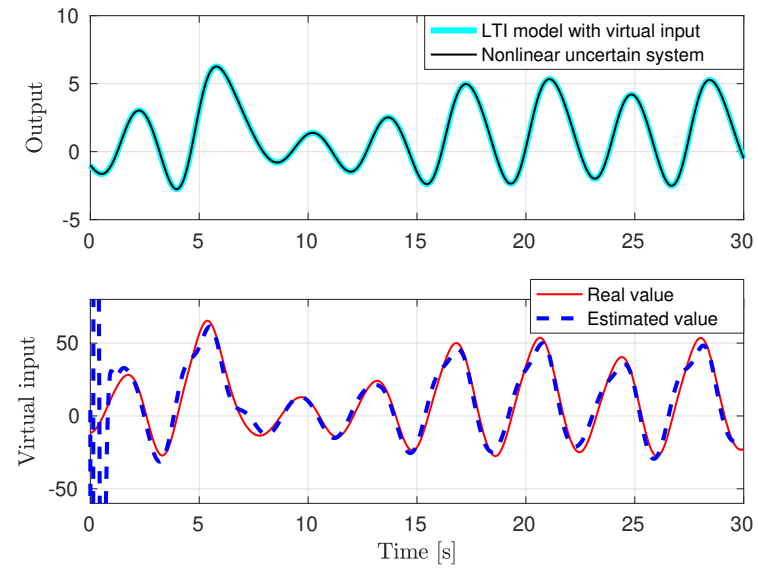

Fig. 1. Evolution of the output $y(t)$ and the virtual input $\mathscr{C}(t)$

Figures 2 and 3 show the estimation error for state components $x_{1}$ and $x_{2}$ respectively, for the different observation schemes. The figure showing the estimation error for the state component $x_{3}$ is omitted for reason of space. However, note that the behavior of the estimation error for $x_{3}$ is similar to the one of $x_{1}$ and $x_{2}$, only exhibiting a transient phase of larger magnitude.

In addition to these figures, let's define the parameter $\Lambda_{k}$ as the upper bound of the estimation error $E_{k}$ on a finite-time window, i.e.:

$$
\Lambda_{k}=\sup _{t \in\left[t_{1}, t_{1}+\tau\right]}\left|E_{k}(t)\right|
$$

where, for relevance, $t_{1}$ has to be chosen larger than the response time of the observer to avoid the transient phase.

TABLE II

$\Lambda_{k}$ ASsociated to $E_{k}$, with $k=1, \ldots, 3$, FOR $i=5$

$\begin{array}{ccc} & \text { ESO / GPI obs. } & \text { GeLESO } \\ E_{1} & 0.0032 & 0.0017 \\ E_{2} & 0.1304 & 0.0700 \\ E_{3} & 2.2785 & 1.1717\end{array}$

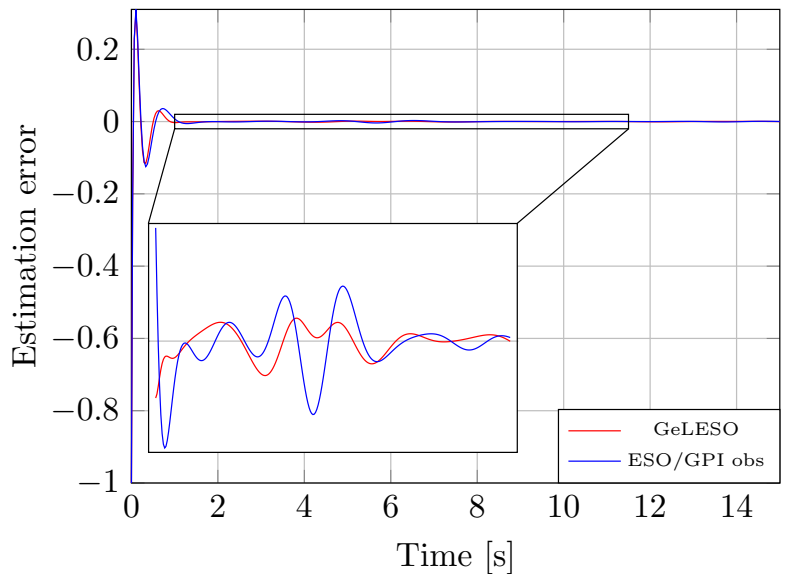

Fig. 2. Estimation errors of the state component $x_{1}(t)$

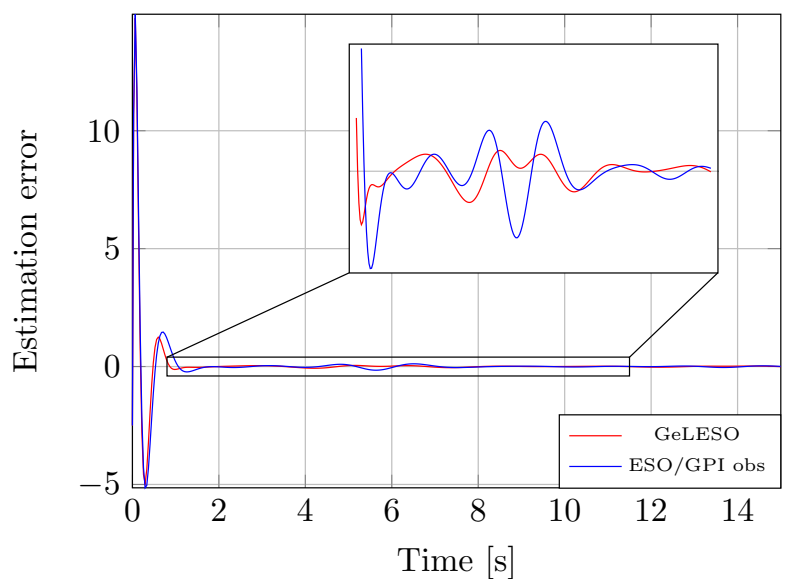

Fig. 3. Estimation errors of the state component $x_{2}(t)$

Table II gives the value of $\Lambda_{k}$ for estimation errors $E_{1}, E_{2}$ and $E_{3}$ of state components $x_{1}, x_{2}$ and $x_{3}$ for each observation scheme, with $t_{1}=20 \mathrm{~s}$ and $\tau=10 \mathrm{~s}$. From figure 2, figure 3 and table II, it is clear that the proposed Generic Linear Extended State Observer provides a more accurate estimation than the higher-order ESO and GPI observers in the literature.

In order to investigate the influence of the number of extended components in the extended state $X_{e}$ (10), table III below gives the value of the parameter $\Lambda_{k}$ for four extended components only, i.e. for $i=4$.

TABLE III

$\Lambda_{k}$ Associated to $E_{k}$, with $k=1, \ldots, 3$, FOR $i=4$

$\begin{array}{ccc} & \text { ESO / GPI obs. } & \text { GeLESO } \\ E_{1} & 0.0060 & 0.0035 \\ E_{2} & 0.2107 & 0.1235 \\ E_{3} & 3.1580 & 1.8509\end{array}$

The first thing to notice is that, when $i=4$, the estimation accuracy dropped for all the observation schemes, hence giving evidence of what was stated in Theorem 2. The number of 
extended components in the extended state $X_{e}$ is therefore an important selection criterion for the design of the observation scheme. The second element to notice is that the GeLESO still provides a more accurate estimation than the higher-order ESO and GPI observers in the literature. Furthermore, comparing tables II and III, one can also notice that the GeLESO with $i=4$ remains close or is even more efficient than the higherorder ESO and GPI observers with $i=5$.

\section{Perspectives}

In addition to the influence of the number $i$ of extended components in the extended state $X_{e}$, the choice of the coefficients $a_{k}$ in (4) also needs to be investigated. Indeed, being able to choose them (as opposed to ADRC where they are all null) asks the question of their usefulness and influence on the observer's performances and future control design. Some preliminary elements on that matter are given in [26], along with the cornerstone of Virtual Input Rejection Control (VIRCO), a control scheme introduced therein.

\section{CONCLUSION}

This paper provides a generic observation methodology that can be applied to a given class of unknown nonlinear SISO systems affected by uncertainties and disturbances. The proposed methodology transforms the state estimation problem of unknown nonlinear SISO systems into the state estimation problem of chosen LTI systems, along with an unknown virtual input. This strategy bypasses any identification step and avoids the mathematical complexity inherent to nonlinear systems. The state and virtual input estimation is performed with a Generic Linear Extended State Observer that is introduced in the paper. This specific observer ingeniously uses the parameters of the chosen LTI system to introduce extended state components that take into account the virtual input and its successive derivatives. Such way to proceed provides a more accurate knowledge of the virtual input and its dynamics. The benefits of the proposed observation scheme are confirmed by numerical simulations involving a Genesio-Tesi chaotic system affected by a non-constant disturbance. Similar benefits are expected with any kind of systems, including applications relying on miniaturized mechatronic devices.

\section{REFERENCES}

[1] R. E. Kalman. A New Approach to Linear Filtering and Prediction Problems, Journal of Basic Engineering, vol. 82, no. 1, 1960.

[2] D. G. Luenberger. Observing the State of a Linear System, IEEE Transactions on Military Electronics, vol. 8, no. 8, pp. 74-80, 1964.

[3] S. Julier and J. Uhlmann. A New Extension of the Kalman Filter to Nonlinear Systems, Proceedings of AeroSense: 11th International Symposium on Aerospace / Defense Sensing, Simulations and Controls, Orlando, FL, USA, 1997.

[4] A. Krener and A. Isidori. Linearization by Output Injection and Nonlinear Observers, Systems \& Control Letters, vol. 3, no. 1, pp. 47-52, 1983.
[5] Y. Dong, H. Wang and Y. Wang. Design of Observers for Nonlinear Systems with $\mathrm{H}_{\infty}$ Performance Analysis, Mathematical Methods in the Applied Sciences, vol.37, no. 5, pp. 718-725, 2013

[6] H. Khalil and L. Praly. High-Gain Observers in Nonlinear Feedback Control, International Journal of Robust and Nonlinear Control, vol. 24, no. 6, pp. 993-1015, 2013.

[7] D. Astolfi. Observers and Robust Output Regulation for Nonlinear Systems, PSL Research University, 2016.

[8] D. Astolfi, L. Marconi, L. Praly and A. R. Teel. Low-Power PeakingFree High-Gain Observers, Automatica, vol. 98, pp. 169-179, 2018.

[9] L. Freidovich and H. Khalil. Performance Recovery of FeedbackLinearization-Based Designs, IEEE Transactions on Automatic Control, vol. 53, no. 10, pp. 2324-2334, 2008.

[10] Z. Gao, Y. Huang and J. Q. Han. An Alternative Paradigm for Control System Design, Proceedings of the 40th Conference on Decision and Control, Orlando, FL, USA, 2001.

[11] Z. Gao. Active Disturbance Rejection Control: A Paradigm Shift in Feedback Control System Design, Proceedings of the American Control Conference, Minneapolis, MN, USA, 2006.

[12] B. Z. Guo and Z. L. Zhao. Active Disturbance Rejection Control for Nonlinear Systems: An Introduction, Wiley, 2016.

[13] J. Q. Han. The Extended State Observer for a Class of Uncertain Systems, Control and Decision, vol. 10, no. 1, pp. 85-88, 1995.

[14] Z. Gao. Scaling and Bandwidth-Parameterization Based Controller Tuning, Proceedings of the American Control Conference, Denver, CO, USA, 2003.

[15] G. Feng, Y. F. Liu and L. Huang. A New Robust Algorithm to Improve the Dynamic Performance on the Speed Control of Induction Motor Drive, IEEE Transactions on Power Electronics, vol. 19, no. 6, pp. 16141627, 2004.

[16] Y. Xia, Z. Zhu, M. Fu and S. Wang. Attitude Tracking of Rigid Spacecraft with Bounded Disturbances, IEEE Transactions on Industrial Electronics, vol. 58, no. 2, pp. 647-659, 2011.

[17] S. E. Talole, J. P. Kolhe and S. B. Phadke. Extended-State-ObserverBased Control of Flexible-Joint System with Experimental Validation, IEEE Transactions on Industrial Electronics, vol. 57, no. 4, pp. 14111419,2010

[18] S. Li, J. Yang, W. H. Chen and X. Chen. Disturbance Observer-Based Control - Methods and Applications, CRC Press, 2014.

[19] J. Wang, S. Li, J. Yang, B. Wu and Q. Li. Extended State ObserverBased Sliding Mode Control for PWM-Based DC-DC Buck Power Converter Systems with Mismatched Disturbances, IET Control Theory \& Applications, vol. 9, no. 4, pp. 579-586, 2015

[20] R. Madoński and P. Herman. Survey on Methods of Increasing the Efficiency of Extended State Disturbance Observers, ISA Transactions, vol. 56, pp. 18-27, 2015.

[21] A. A. Godbole, J. P. Kolhe and S. E. Talole. Performance Analysis of Generalized Extended State Observer in Tackling Sinusoidal Disturbances, IEEE Transactions on Control Systems Technology, vol. 21, no. 6, pp. 2212-2223, 2013.

[22] R. Madoński and P. Herman. On the Usefulness of Higher-Order Disturbance Observers in Real Control Scenarios based on Perturbation Estimation and Mitigation, Proceedings of the 9th International Workshop on Robot Motion and Control, Kuslin, Poland, 2013.

[23] A. Luviano-Juárez, J. Cortés-Romero and H. Írez. Synchronization of Chaotic Oscillators by Means of Generalized Proportional Integral Observers, International Journal of Bifurcation and Chaos, vol. 20, no. 5, pp. 1509-1517, 2010.

[24] H. Sira-Ramírez, A. Luviano-Juárez, M. Ramírez-Neria and E. W. Zurita-Bustamante. Active Disturbance Rejection Control of Dynamic Systems - A Flatness Based Approach, Butterworth-Heinemann, 2017.

[25] Q. Zheng, L. Q. Gao and Z. Gao. On Stability Analysis of Active Disturbance Rejection Control for Nonlinear Time-Varying Plants with Unknown Dynamics, Proceedings of the 46th Conference on Decision and Control, New Orleans, LA, USA, 2007.

[26] F. Amokrane, E. Piat, J. Abadie, A. Drouot and J. Escareno. State Observation of Unknown Nonlinear SISO Systems based on Virtual Input Estimation, International Journal of Control, in press, DOI 10.1080/00207179.2019.1680869, 2019. 